\title{
Role of Convalescent Plasma Therapy in Patients with Moderate to Severe COVID 19
}

\author{
Khurshid Ahmed Khan, Muhammad Kamran Rauf, \\ Asma Afzal Khan, Javeid Iqbal, Sadia Taj, Fatima Shahid, \\ Aijaz Zeeshan Khan Chachar
}

\begin{abstract}
Significance: Given the lack of evidence of effective treatments and vaccines for Corona virus disease19(COVID-19), several treatment options are under investigation, including Convalescent Plasma. The FDA granted emergency use authorization (EUA) on August 23, 2020 for use of convalescent plasma in hospitalized patients with COVID-19 but further data from randomized clinical trials are needed. Objective: The objective of the study was to find evidence of efficacy of Convalescent Plasma therapy in patients with moderate to severe COVID-19. Study Design, Setting, and Sample size: Open label, observational study, where participants were allocated using predefined assessment criteria. The study included 42 patients admitted in Fatima Memorial Hospital, Lahore with moderate to severe COVID-19. The study was terminated early after 42 patients of a planned sample size of 50 patients due to no further admissions of COVID-19 in the hospital. Groups: Standard treatment for COVID-19 disease (antibiotics, steroids, Tocilizumab, Remdesivir) according to disease severity was given in 15 patients (control group), while Convalescent plasma was administered in addition to standard treatment in 27 patients (cases). Primary and Secondary Outcomes: Primary outcome was to see clinical improvement in patients with moderate to severe COVID-19, defined as patient recovered and discharged home. Secondary outcomes looked at oxygen requirement in both cases and controls after administration of treatment and any improvement in biochemical markers. Results: 42 patients were observed in the study. 27 patients assigned to the cases group received standard treatment and convalescent plasma therapy for moderate to severe Covid-19 disease whilst 15 patients received standard treatment only, assigned as control group. The median age of patients in the convalescent plasma group was $59.81 \pm 10.14$ and $54.37 \pm 12.93$ in the control group ( $\mathrm{p}$-value $=0.086$ ). There was no statistically significant difference in baseline characteristics between the cases and control group. Primary outcome measured as patient recovered and discharged home was achieved in $64.3 \%$ of patients in the cases group and $35.7 \%$ in the control group ( $\mathrm{p}$ value $=1$ ). Secondary outcome compared oxygen requirements in cases (median $201 / \mathrm{min}$ ) and controls (median $8 \mathrm{l} / \mathrm{min}$ ) after treatment. There was no statistically significant difference in oxygen requirements between cases and controls after treatment $(\mathrm{p}=0.059)$. Biochemical markers of inflammatory response such as Total leukocyte count, D-dimer, Lactate dehydrogenase (LDH) did not show any statistically significant difference in the two groups. CRP however decreased markedly in those patients treated with Convalescent plasma in addition to standard treatment when compared with control group ( $\mathrm{p}$ value=0.002). Also, serum Ferritin was also found to be much lower in cases as compared to controls ( $p$ value $=0.001$ ). No adverse effects were seen in the cases after administration of convalescent plasma. Conclusion: Convalescent Plasma therapy administered in addition to standard treatment did not result in a statically significant improvement in patient survival in patients with moderate to severe COVID-19 when compared with standard treatment alone. There was however, a significant reduction in two inflammatory markers associated with severe disease i.e. C-reactive protein (CRP) and serum Ferritin in the cases group. The study results may have failed to detect a clinically significant difference because of early termination of the study resulting in small sample size.
\end{abstract}

Keywords: Covid-19, SARS-CoV-2, Corona Virus, Convalescent Plasma Therapy

\section{Introduction:}

The end of 2019 saw the spread of a novel corona virus identified as a cause of a cluster of pneumonia cases in Wuhan, a city in Hubei province of China. This virus, named as acute respiratory syndrome corona virus 2(SARS-CoV-2) was the cause of the disease, COVID-19. The virus transmitted from respiratory droplets, quickly spread around the world causing a global pandemic.

\begin{abstract}
Several treatments which are already in use for other clinical indications are under investigation for COVID-19. These include anti-viral drugs such as Lopinavir, Ritonivir, Remdesivir and immunomodulatory drugs such as Tocilizumab. Remdesivir is a novel nucleotide analogue that has activity against severe acute respiratory syndrome coronavirus 2 (SARS-CoV-2) in vitro. ${ }^{2}$
\end{abstract}

This article is published under the terms of the Creative Commons Attribution License 4.0 Author(s) retain the copyright of this article. Publication rights with Alkhaer Publications. Published at: http://www.ijsciences.com/pub/issue/2020-12/

DOI: 10.18483/ijSci.2423; Online ISSN: 2305-3925; Print ISSN: 2410-4477 
Several observational studies, and randomized control trials are underway evaluating the efficacy of Tocilizumab, an interleukin-6(IL-6) receptor inhibitor. This immunomodulatory drug already used for rheumatic diseases and cytokine release syndrome is being investigated for treatment of severe COVID19.

Currently, there is little evidence for effective treatments of COVID-19 and vaccines are under trial. Several treatments are under investigation which have previously been used in pandemics, including the Spanish flu, SARS-Co V, West Nile virus, and more recently, Ebola virus. ${ }^{3,4,5,6}$ Passive immunization has been used in prevention and management of infectious diseases since early 20th century ${ }^{3}$. This includes Convalescent Plasma, a passive immune therapy which requires administration of specific antibodies against the agent for treating an infectious disease caused by that agent. Convalescent plasma is obtained using apheresis from patients recovered from the disease. Mechanism of action is viral neutralisation ${ }^{2}$. Most observational studies to date suggest administration of convalescent plasma $(\mathrm{CP})$, preferably with high antibody titer, in early disease, prior to development of life-threatening inflammatory disease.

During apheresis, in addition to neutralizing antibodies (NAbs), other proteins such as antiinflammatory cytokines, clotting factors, natural antibodies, defensins, pentraxins and other undefined proteins are obtained from donors ${ }^{7}$.In this sense, transfusion of $\mathrm{CP}$ to infected patients may provide further benefits such as immunomodulation via amelioration of severe inflammatory response ${ }^{8}$. The latter could be the case of COVID-19 in which an over-activation of the immune system may come with systemic hyperinflammation or "cytokine storm" driven by IL-1 $\beta$, IL-2, IL-6, IL-17, IL-8er, TNF $\alpha$ and CCL2. This inflammatory reaction may perpetuate pulmonary damage entailing fibrosis and reduction of pulmonary capacity ${ }^{9,10}$.

In the United States, the Food and drug administration (FDA) is accepting investigational new drug applications for use of convalescent plasma for patients with severe or life-threatening COVID19 .One case series described administration of plasma from donors who had completely recovered from COVID-19 to five patients with severe COVID19 on mechanical ventilation and persistently high viral titers despite investigational antiviral treatment $^{11}$. The patients had decreased nasopharyngeal viral load, decreased disease severity score, and improved oxygenation by 12 days after transfusion ${ }^{11}$. However, these findings do not establish a causal effect, and the efficacy of convalescent plasma remains unknown

The convalescent donors must undergo standard predonation assessment to ensure compliance with current regulations regarding plasma donation ${ }^{12}$. Currently, convalescent donors between 18 and 65 are considered as subjects without infectious symptomatology and a negative test for COVID-19 after 14 days of recovery. These tests must be repeated $48 \mathrm{~h}$ later and at the moment of donation ${ }^{12}$. Apheresis is the recommended procedure to obtain plasma. This procedure is based on a continuous centrifugation of blood from donor to allow selective collection plasma. The efficiency of this technique is around $400-800 \mathrm{~mL}$ from a single apheresis donation. This amount of plasma could be storage in units of 200 or $250 \mathrm{~mL}$, and frozen within $24 \mathrm{~h}$ of collection to be used in further transfusions ${ }^{13}$.

We want to know whether plasma from people who have recovered from COVID-19 is an effective treatment for people with COVID-19 in our geography, and whether this treatment causes any unwanted effects.

\section{Methods: \\ Study design:}

It was an open label, observational study, where participants were allocated using predefined protocol. Those patients who met the inclusion criteria of study after signing an informed consent either by the patient or the person legally authorized to sign were included. Patients not receiving convalescent plasma therapy were considered as control and were given standard available treatment as approved by FDA and local authorities. Standard treatment included giving antibiotics, steroids (as recommended by the Recovery Trial, United Kingdom,UK), Tocilizumab (for patients in cytokine storm) and Remdesivir (antiviral medication).

\section{Sample size:}

Our sample size was aimed at 50 patients calculated by the results from previous study which showed improvement in $80 \%$ of patients. However due to diminished cases of COVID-19 in the region, no further admissions were done and the study was terminated.

\section{Sampling technique:}

Our sampling technique was probability sampling according to inclusion and exclusion criteria.

\section{Duration of study:}

The study was conducted over a three month period, from May 2020 till July 2020.

\section{Study settings:}

Study was conducted at Fatima Memorial 
Hospital,FMH COVID-19 hospitalized patients.

\section{Donors Eligibility Criteria:}

1. All the regulations related to International Conference on Harmonization guideline for good clinical practice (ICH-GCP) should be followed.

2. Selected donors are to be in good health fulfilling the criteria of a healthy blood donor.

3. Donor should have a documented prior diagnosis of Covid-19 by RT-PCR from the Laboratories authorized by Government of Pakistan for Covid-19 testing.

4. Donor should be symptom free for a minimum of 14 days.

5. Viral RNA detection by reverse transcriptasepolymerase chain reaction (RT-PCR) from nasopharyngeal/oral-pharyngeal swab specimens should be negative. (The negative RT-PCR report should be from the Laboratories authorized by Government of Pakistan for Covid-19 testing).

6. Donor should donate at least 14 days after negative RT-PCR.

7. At least 7 days have passed since last use of steroids.

8. Age cutoff: $18-60$ year

9. Body weight $>50 \mathrm{~kg}$

10. To avoid the risk of transfusion related acute lung injury (TRALI) and Human Leucocytes antigen (HLA) antibodies, plasma should be extracted preferably from male donors or from nulliparous females.

11. Informed consent (attached) was obtained from all donors.

\section{Inclusion Criteria (recipient):}

(Informed consent will be taken from all potential patients)

1. Age minimum 18 years

2. confirmed COVID -19 cases PCR positive

3. Pneumonia confirmed by chest imaging

4. Moderate severe or severe COVID 19 features a. shortness of breath oxygen saturation $<93 \%$ on room air

b. Respiratory rate $>30 / \mathrm{min}$

c. Partial pressure of arterial oxygen to fraction of inspired oxygen ratio $<300$

d. Lung infiltrates on chest $\mathrm{X}$ $\operatorname{ray}(\mathrm{CXR})>50 \%$ within 24 to $48 \mathrm{hrs}$

e. Respiratory failure

\section{Exclusion criteria(recipient):}

1. Critical COVID, defined by
a. Shock
b. Multi Organ Failure(MOF)
c. Severe congestive heart failure (Pre- COVID 19 Ejection fraction (EF) $<30 \%$ )
d. Chronic renal failure on maintenance haemodialysis
e. Decompensated Chronic Liver disease
f. Age less than 18 or more than 70 with comorbidities
g. Pregnant females

\section{CP infusion protocols:}

Patients will receive an initial dose of $200 \mathrm{~mL}$, followed by one or two additional doses of $200 \mathrm{~mL}$ according to disease severity and tolerance of the infusion. Predefined Parameters of disease severity will be assessed 12 to 24 hourly.

\section{Standard Treatment:}

Standard treatment was given to all patients and included anti-bacterial medication (Azithromycin for all patients and Meropenem for secondary infections), steroids (dexamethasone, methylprednisolone), anti-coagulants (enoxaparin, heparin), Tocilizumab (for patients in cytokine storm) and anti-viral medication (Remdesivir). This was provided based on latest national guidelines and hospital protocols.

\section{Outcome Measures:}

The primary endpoint of the study was patient recovery and discharge home. Secondary outcomes assessed the response in patients who received convalescent plasma compared with patients receiving standard treatment.

Assessment of response in patient was measured using following parameters:

1 .Clinical parameters:

a. Improvement in oxygen saturation

b. Decrease oxygen requirements

c. Weaning of from ventilator support

d. Normalization of body temperature

\section{2 .Laboratory parameters:}

a. Improvement in inflammatory markers such as D-Dimers, CRP \& serum Ferritin levels.

b. PCR negative

\section{3 .Radiological parameter:}

Chest $\mathrm{x}$ ray showing decreasing infiltrates more than $50 \%$ improvement from base line.

\section{Statistical Analysis:}

Baseline demographic data such as clinical symptoms, co-morbidities were presented as mean and percentages. Variables including clinical and laboratory parameters were presented as the median and IQR. Statistical software used included SPSS statistics version 20.0. Statistical test applied was Mann-Whitney Test. Comparison charts and graphs were plotted using Microsoft Word 2007 and Microsoft Excel 2007. 


\section{Results:}

A total of 42 patients were observed in the study. 27 of these patients received standard treatment and convalescent plasma therapy (cases) for moderate to severe Covid-19 disease whilst 15 received standard treatment, assigned as control group.
The median age of patients in the convalescent plasma group was $59.81 \pm 10.14$ and $54.37 \pm 12.93$ in the control group $(\mathrm{P}$ value $=0.086)$. There was no statistically significant gender difference between the convalescent plasma group and the control group ( $p$ value $=0.734)$. Table- $1 \&$ Figure- 1

\begin{tabular}{|ll|l|l|l|}
\hline Variables & Cases & Controls & p-value \\
\hline Age & $59.81 \pm 10.14$ & $54.37 \pm 12.93$ & 0.086 \\
\hline Gender & Male & $17(63)$ & $11(73.3)$ & 0.734 \\
\\
\hline \multicolumn{2}{|l|}{ Female } & $10(37.3)$ & $4(26.7)$ & \\
\end{tabular}

Table \&Figure 1: Age and gender distribution

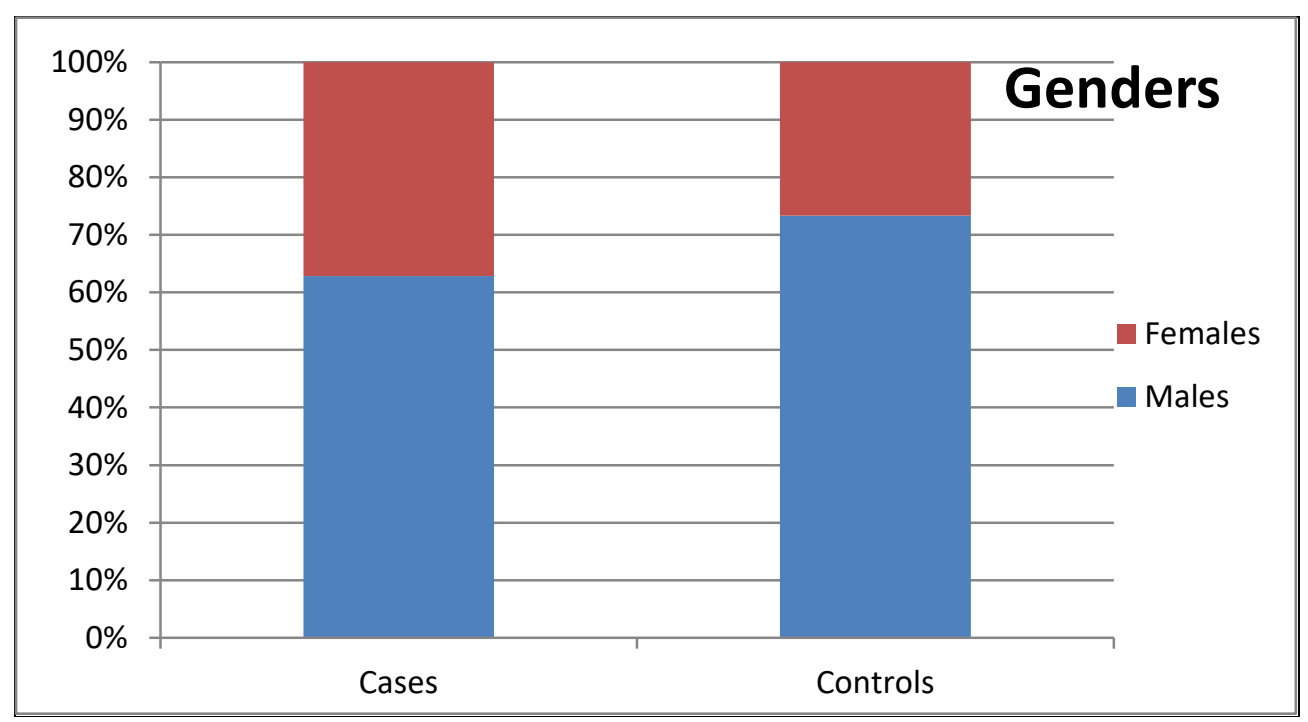

The commonest symptoms in both groups were cough, fever and dyspnea. $70.4 \%$ (19) patients in the convalescent plasma group and $80 \%(12)$ patients in the control group ( $\mathrm{p}$ value $=0.717$ ) had cough on admission. $88.9 \%(24)$ patients in the convalescent plasma group had fever while $73.3 \%(11)$ patients presented with fever on admission ( $\mathrm{p}$ value $=0.225$ ). Dyspnea, the commonest presenting symptom, with
92.6\%(25) of patients in convalescent plasma group presenting with shortness of breath on admission. 93.3\%(14) of the control group presented with dyspnea ( $\mathrm{p}$ value=1.000). Other symptoms such as diarrhea, nausea, vomiting, myalgia, anosmia, and loss of taste were seen equally in both groups with no statistical difference. Table 2 and figure 2

Table-2: Presenting symptoms

\begin{tabular}{|l|l|l|l|}
\hline & Cases & Controls & P - value \\
\hline Cough & $19(70.4)$ & $12(80)$ & 0.717 \\
\hline Fever & $24(88.9)$ & $11(73.3)$ & 0.225 \\
\hline Dyspnea & $25(92.6)$ & $14(93.3)$ & 1.000 \\
\hline Nausea & $1(3.7)$ & $1(6.7)$ & 1.000 \\
\hline Vomiting & $1(3.7)$ & $0(0)$ & 1.000 \\
\hline Diarrhoea & $4(14.8)$ & $1(6.7)$ & 0.639 \\
\hline Myalgia & $2(7.4)$ & $2(13.3)$ & 0.608 \\
\hline Anosmia & $2(7.4)$ & $0(0.0)$ & 0.530 \\
\hline Loss of taste & $3(11.1)$ & $0(0.0)$ & 0.541 \\
\hline
\end{tabular}




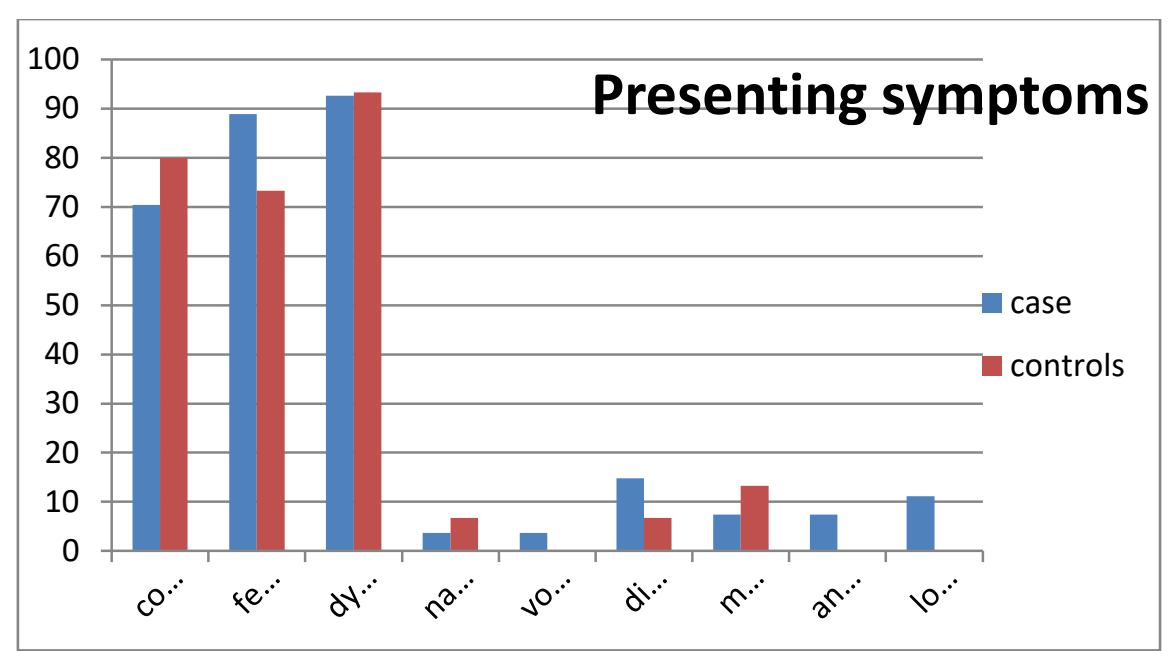

Figure: 2: Presenting symptoms in convalescent plasma group (case) and standard treat group(control)

Table-3: Comorbidities in Case and Control group

\begin{tabular}{|l|l|l|l|}
\hline Co-morbidities & Cases & Controls & p-value \\
\hline Diabetes & $17(63)$ & $7(46.7)$ & 0.347 \\
\hline Hypertension & $20(74.1)$ & $5(33.3)$ & 0.020 \\
\hline Cardiovascular Disease & $6(22.2)$ & $1(6.7)$ & 0.390 \\
\hline Chronic Kidney Disease & $2(7.4)$ & $1(6.7)$ & 1.000 \\
\hline Obesity & $7(25.9)$ & $0(0.0)$ & 0.038 \\
\hline Lung Disease & $5(18.5)$ & $2(13.3)$ & 1.000 \\
\hline
\end{tabular}

Hypertension was the most prevalent co-morbidity with $74.1 \%$ (20) patients in the convalescent plasma group and $33.3 \%$ (5) patients in the control group known to have the disease. Diabetes, the second most prevalent condition was found in $63 \%$ (17) patients), while $46.7 \%$ (7) patients suffered from diabetes in the control group, $\mathrm{p}$ value $=0.347$. A total of 6 patients $(22.2 \%)$ had a history of cardiovascular disease in the convalescent plasma group while 1 patient $(6.7 \%)$ was known to have cardiovascular disease in the control group. Chronic kidney disease was found in $7.4 \%$ of patients in convalescent plasma group and $6.7 \%$ in the controls. Although, 7 patients in the convalescent plasma group were obese, none in the control were obese. However, this difference was not found to be statistically significant as $\mathrm{p}$ value was 0.038. The most common lung disease was Asthma. Patients with known history of lung disease were equally distributed in both groups. Table-3 \& Figure3

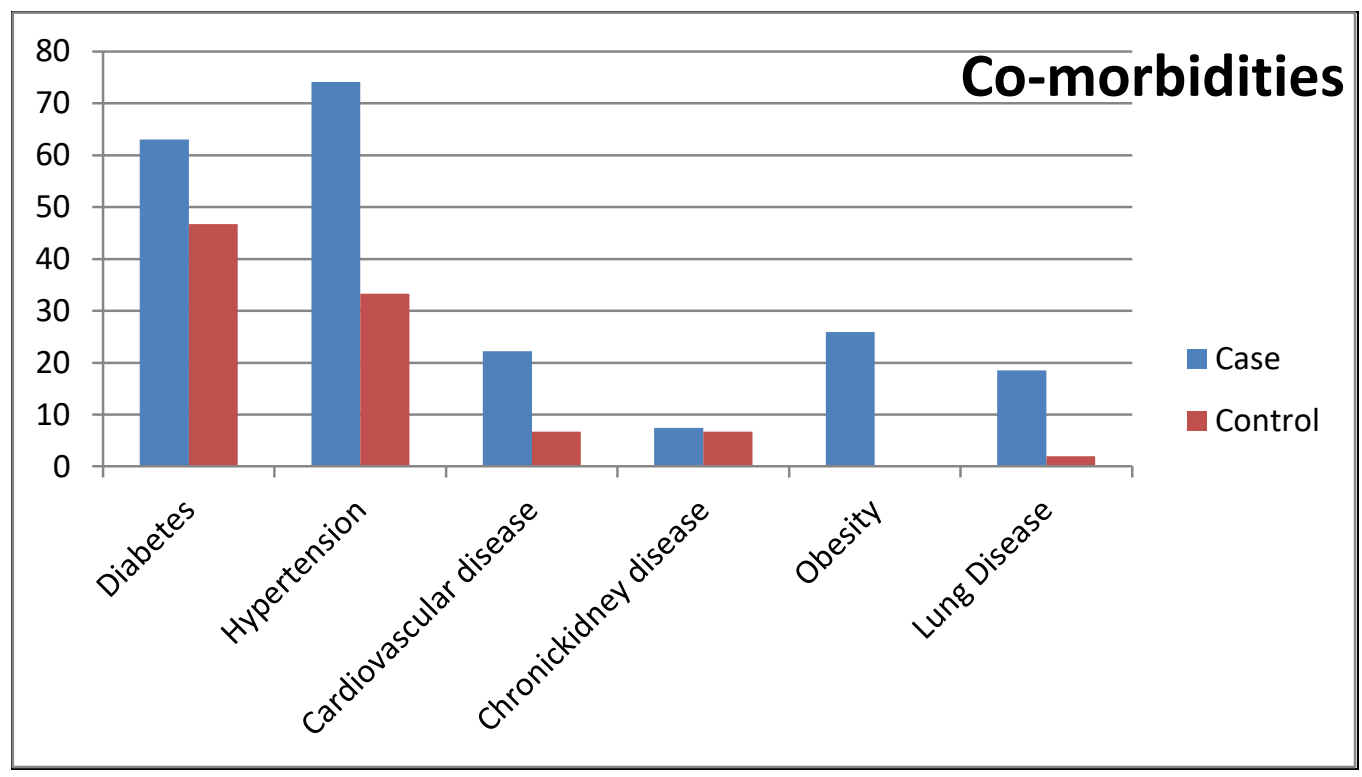

Figure: 3: Co-morbidities in convalescent plasma group (case) and standard treat group(control) 
No statistically significant differences were found in baseline demographics of the cases and controls group.

Secondary outcome included reviewing patient's oxygen requirement and oxyhaemoglobin saturation, $\mathrm{SaO} 2$. Oxygen requirement was defined as the minimum amount of oxygen required to keep
$\mathrm{SaO} 2>94 \%$. Patient's oxygen requirements were measured on a daily basis before and after treatment. All patients required oxygen on admission (minimum oxygen requirement was $2 \mathrm{~L}$ and maximum was $15 \mathrm{~L}$ ). The oxygen requirement increased with disease progression. However, there was no significant difference in reduction of oxygen requirements between cases and controls. Graph-1.

\begin{tabular}{|l|l|l|l|l|}
\hline Variable & & Case & Control & P-Value \\
\hline \multirow{3}{*}{ Oxygen requirement } & On admission & $5(5)$ & $10(10)$ & 0.01 \\
\cline { 2 - 5 } & $\begin{array}{l}\text { After treatment } \\
\text { (day1) }\end{array}$ & $20.00(5.00)$ & $8.00(11.00)$ & 0.059 \\
\hline
\end{tabular}

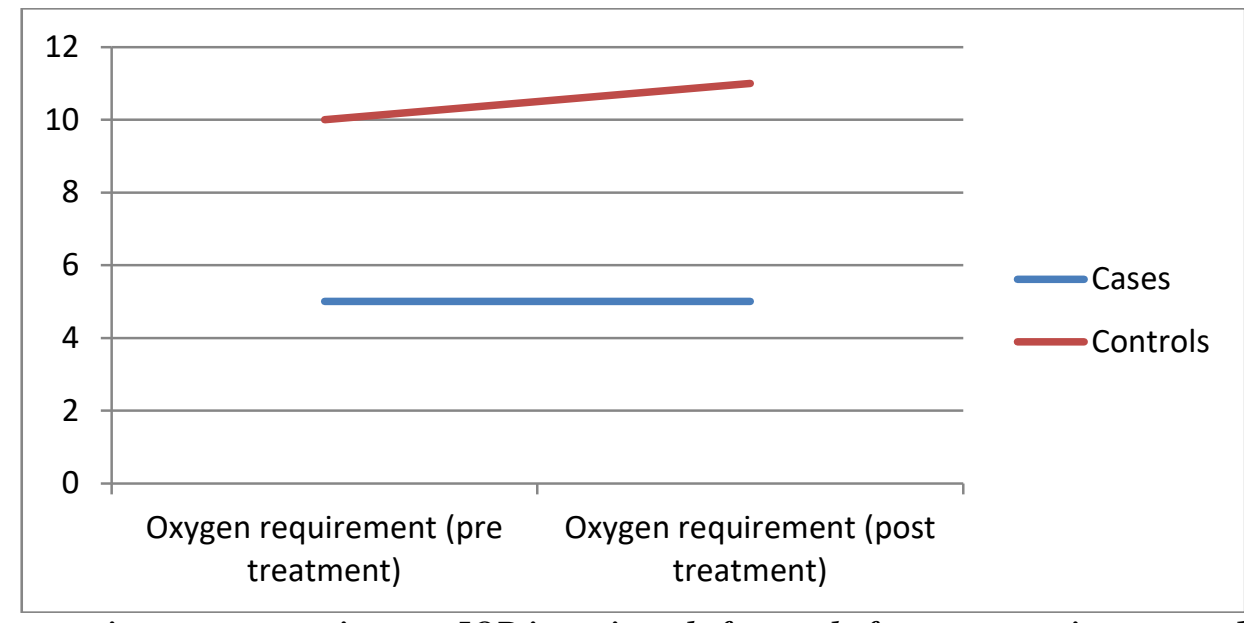

Graph:1: Representing oxygen requirement IQR in patients before and after treatment in cases and controls.

The assessment of response in patient was also measured using laboratory parameters mainly inflammatory markers such as D-Dimers, LDH, CRP $\&$ serum Ferritin levels. Total leukocyte count was measured for any secondary bacterial infections. There was no statistical difference in the laboratory parameters such as TLC, d-dimers, CRP, LDH, Creatinine and ALT between the cases and controls. Serum Ferritin was higher (median 1074(1285.75)) in the control group versus cases (median 636(327) pvalue $=0.004$. A reduction of serum Ferritin was noted in both groups after treatment 636(327) to 456 (128) versus $1074(1285.75)$ to $950(1118.25)$, pvalue $=0.001$. The results demonstrated that after administration of convalescent plasma there was a reduction in $\mathrm{C}$-reactive protein from median $129(284.25)$ to median 30(52.30). This difference was not shown in the controls where C-reactive protein from median 98(163.00) did not significantly change, median 139.50(144.05). Difference between cases and controls after treatment was significant with p-value $=0.002$. Table- 4 \& Graph 2 and 3.

\begin{tabular}{|c|c|c|c|c|}
\hline & Variables & Case & Control & P-Value \\
\hline \multirow[t]{7}{*}{ Pre } & Total Leukocyte Count & $11(7)$ & $10.8(4.98)$ & 0.906 \\
\hline & D-dimer & $1.2(445.10)$ & $1.1(1.50)$ & 0.348 \\
\hline & CRP & $129(284.25)$ & $98(163.00)$ & 0.172 \\
\hline & Ferritin & $636(327)$ & $1074(1285.75)$ & 0.004 \\
\hline & $\mathrm{LDH}$ & $378(225.75)$ & $497(541)$ & 0.180 \\
\hline & Creatinine & $0.80(0.58)$ & $0.91(0.30)$ & 0.177 \\
\hline & ALT & $40(19)$ & $49(24)$ & 0.103 \\
\hline \multirow[t]{7}{*}{ Post } & Total Leukocyte Count & $11(8.43)$ & $10.32(2.28)$ & 0.601 \\
\hline & D-dimer & $1.00(2.90)$ & $1.10(1.84)$ & 0.579 \\
\hline & CRP & $30(52.30)$ & $139.50(144.05)$ & 0.002 \\
\hline & Ferritin & $456(128)$ & $950(1118.25)$ & 0.001 \\
\hline & LDH & $310(196)$ & $429(529.25)$ & 0.475 \\
\hline & Creatinine & $0.80(1.10)$ & $0.90(0.56)$ & 0.239 \\
\hline & ALT & $43(21)$ & $44.50(19.50)$ & 0.141 \\
\hline
\end{tabular}

Table:4 Pre and Post therapy biochemical markers analysis, represented as median and IQR, interquartile range 


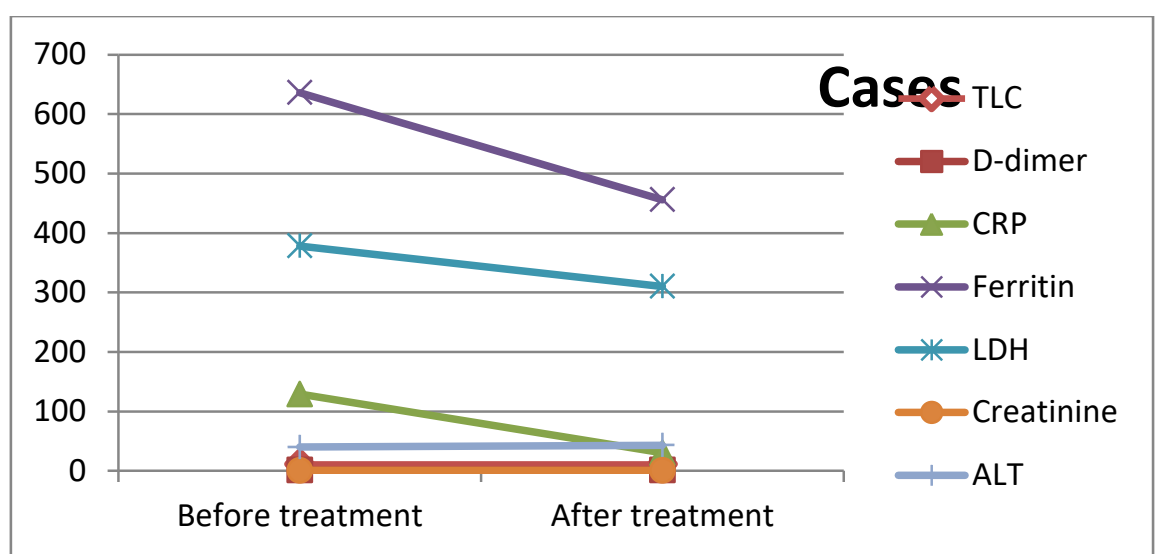

Graph:2-Depicts the trend in inflammatory markers before and after treatment with convalescent plasma along with standard treatment.

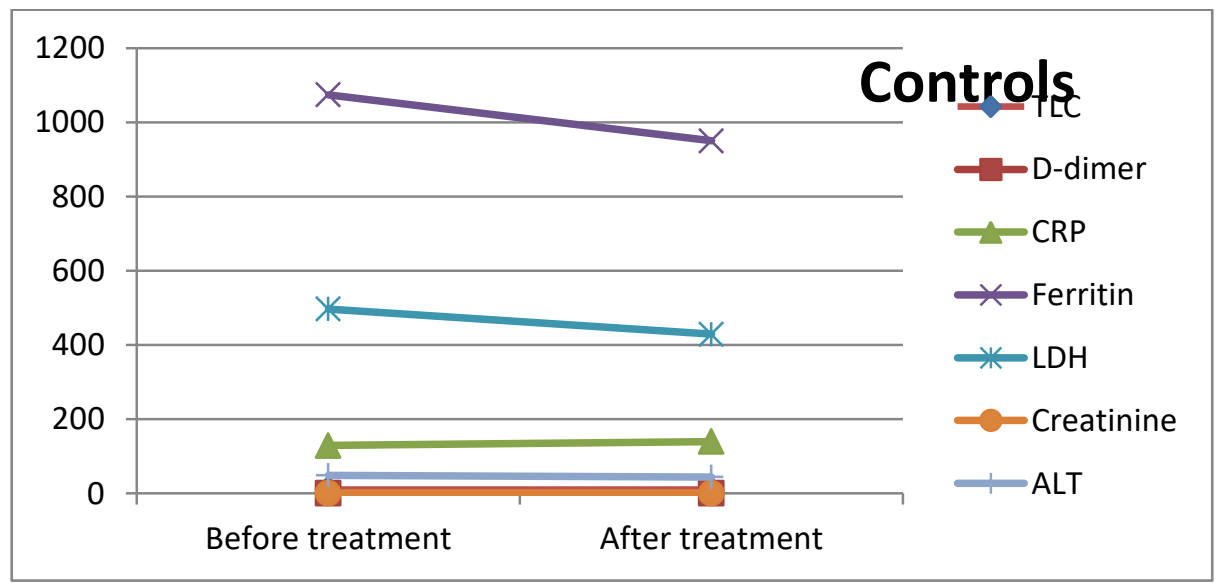

Graph: 3- Depicts the trend in inflammatory markers in patients who received standard treatment.

The primary end point was patient recovery and discharge home. The discharge criteria was based on normalization of body temperature, resolution of presenting symptoms, maintaining oxygen saturation $>94 \%$ at room air at rest and a repeat PCR negative and chest $\mathrm{x}$ ray showing decreasing infiltrates more than $50 \%$ improvement from base line.
The rate of recovery in the two groups was $64.3 \%$ versus $35.7 \% \quad(\mathrm{p}$-value $=1)$ signifying that convalescent plasma did not improve patient survival. No adverse events were reported after the transfusion of convalescent plasma.Figure-4.

\begin{tabular}{|l|l|l|l|}
\hline & Cases & Controls & P value \\
\hline Recovery Rate & 81.5 & 80 & 1 \\
\hline Mortality Rate & 18.5 & 20 & 1 \\
\hline
\end{tabular}

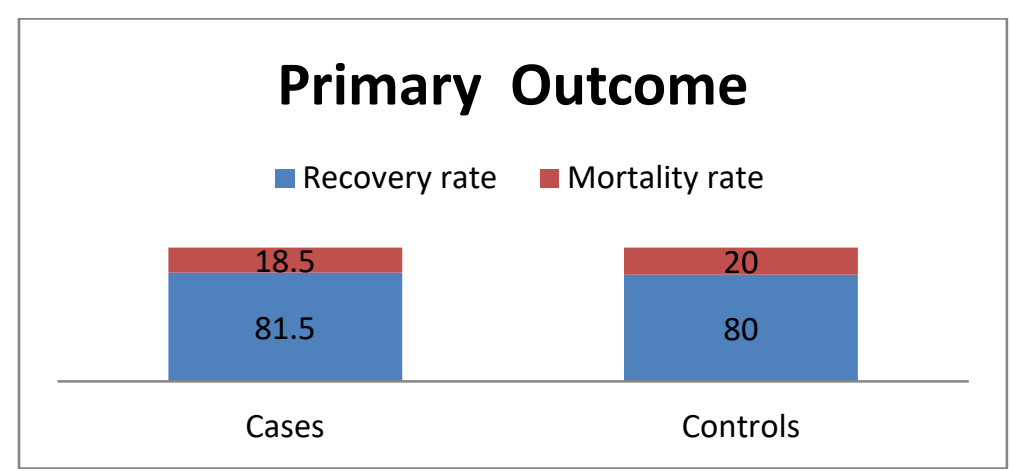

Figure-4: Shows the recovery rate in cases and controls. 


\section{Discussion:}

According to the official WHO Corona virus (Covid19) Dashboard, the number of confirmed COVID-19 cases stand at 37,109,851 including 1,070,355 deaths. This number is rising and the general public seems to be in a state of panic since there is no vaccine for its treatment as of yet. Scientists have little to work on as this is a new and unknown virus.

According to this official website, total number of Covid-19 cases in Pakistan has reached around 318,932. Total death toll has risen to a dangerous high of 6570, with signs of a second wave emerging. Data suggests that $15 \%$ of patients who get infected with SARS COV 2 develop severe infection, requiring oxygen, while $5 \%$ get critical infection, requiring ventilation. Therefore, it is imperative that a solution is sought at the earliest for the treatment of large numbers of these moderate to critically ill patients.

$\mathrm{CP}$ is a safe and potentially effective strategy for the treatment of emerging and re-emerging pathogens, especially in those scenarios without proved antiviral agents or vaccines. Intravenous immunoglobulins (IVIG) and CP shared similar mechanisms of action. The potential antiviral and immunomodulatory effects of $\mathrm{CP}$ are currently being evaluated in COVID-19. Our research aimed at finding the efficacy of Convalescent Plasma in moderate to severe Covid-19. The initial experimental use of CP in Korea* and Wuhan, China* involved administering $\mathrm{CP}$ to 2 patients and 10 patients respectively. Both trials reported a favorable outcome with improvement in biochemical and clinical parameters. This prompted further research with multi-centered, randomized control trials being conducted in different countries around the world. A large multi-centered RCT recruiting 103 patients* was conducted in Wuhan, China, and the PLACID trial* $^{*}$ (open-label parallel-arm phase II multicentre randomized controlled trial conducted in India. Both trials did not indicate any reduction in mortality. Our research data also suggested that convalescent plasma therapy did not add any survival benefit in this patient cohort when compared with current standard treatment. This is contrary to the some of the results of the secondary end point, where a clear improvement in some of the biochemical markers such as CRP, Serum Ferritin is seen. The remaining biochemical markers suggest a decline in the inflammatory process and improvement in patient oxyhaemoglobin Saturation, $\mathrm{SaO} 2$ irrespective of the administration of convalescent plasma.

Our study had its limitations as it was only a single centre study restricted to a specific group of people. We were also unable to attain our sample size of 50 participants due to a reduction in number of patients admitted with severe SARS COV2. There were also confounding variables such as administration of other immunomodulatory drugs such as Tocilizumab for cytokine storm and use of steroids which are known to be effective in moderate to severe COVID 19 disease (Recovery Trial)*. The anti-viral drug, Remdesivir was given to ten patients. Remdesivir has been found to improve recovery rate in patients when compared with placebo*.

Our study failed to demonstrate any improvement in survival when compared with standard treatment. Our recommendations based on the study are that Convalescent plasma is safe therapeutic option and may have a role when administered early in the viral replication stage (first 14 days) especially when other treatments may be contraindicated, as it demonstrated reduction in inflammatory markers.

\section{References:}

1. 1.G. Marano, S. Vaglio, S. Pupella, G. Facco, L. Catalano, G. M. Liumbruno, et al.Convalescent plasma: New evidence for an old therapeutic tool?

2. Blood Transfus, 14 (2016), pp. 152-157, 10.2450/2015.013115

3. T. Burnouf, J. SeghatchianEbola virus convalescent blood products: Where we are now and where we may need to go

4. TransfusApher Sci, 51 (2014), pp. 120 125, 10.1016/j.transci.2014.10.003

5. J. Mair-Jenkins, M. Saavedra-

Campos, J.K. Baillie, P. Cleary, F.-M. Khaw, W.S. Lim, et al.The effectiveness of convalescent plasma and hyperimmune immunoglobulin for the treatment of severe acute respiratory infections of viral etiology: A systematic review and exploratory meta-analysis

6. J Infect Dis, 211 (2015), pp. 80-90, 10.1093/infdis/jiu396

7. M. Rojas, D.M. Monsalve, Y. Pacheco, Y. AcostaAmpudia, C. Ramírez-Santana, A.A. Ansari, et al.Ebola virus disease: An emerging and re-emerging viral threat

8. J Autoimmun, 106 (2020), p. $102375,10.1016 /$ j.jaut.2019.102375

9. C.B. Planitzer, J. Modrof, T.R. KreilWest Nile virus neutralization by US plasma-derived immunoglobulin products

10. J Infect Dis, 196 (2007), pp. 435-440, 10.1086/519392

11. M. Haley, A.S. Retter, D. Fowler, J. Gea-

Banacloche, N.P. O'GradyThe role for intravenous immunoglobulin in the treatment of West Nile virus encephalitis Clin Infect Dis, 37 (2003), pp. e88e90, 10.1086/377172

12.

O. Garraud, F. Heshmati, B. Pozzetto, F. Lefrere, R. Girot, A. Saillol, et al.Plasma therapy against infectious pathogens, as of yesterday, today and tomorrow

$\begin{array}{lll}\text { 13. Transfus Clin } \quad \text { Biol, } 23 \text { (2016), } & \text { pp. }\end{array}$ 44, 10.1016/j.tracli.2015.12.003

14.

O. Garraud, F. Heshmati, B. Pozzetto, F. Lefrere, R. Girot, A. Saillol, et al.Plasma therapy against infectious pathogens, as of yesterday, today and tomorrow

15. Transfus Clin Biol, 23 (2016), pp. 3944, 10.1016/j.tracli.2015.12.003

16. J.D. Lünemann, F. Nimmerjahn, M.C. DalakasIntravenous immunoglobulin in neurology--mode of action and clinical efficacy

17. Nat Rev Neurol, $11(2015), \quad$ pp. 8089, 10.1038/nrneurol.2014.253

18. Dis. doi:10.1093/infdis/jiaa228 
19. D. McGonagle, K. Sharif, A. O'Regan, C. BridgewoodThe role of cytokines including interleukin-6 in COVID-19 induced pneumonia and macrophage activation syndromelike disease

20. Autoimmun Rev (2020), 10.1016/j.autrev.2020.102537

21. In press: 102537

22. Zeng QL, Yu ZJ, Gou JJ, et al. Effect of convalescent plasma therapy on viral shedding and survival in COVID-19 patients [published online April 29, 2020]. J Infect

23. P. Tiberghien, X. deLambalerie, P. Morel, P. Gallian, K. Lac ombe, Y. YazdanpanahCollecting and evaluating convalescent plasma for COVID-19 treatment: Why and how

24. Vox Sang (2020), 10.1111/vox.12926

25 .

E.M. Bloch, S. Shoham, A. Casadevall, B.S. Sachais, B. Shaz , J.L. Winters, et al.Deployment of convalescent plasma for the prevention and treatment of COVID-19

26. J Clin Invest (2020), 10.1172/JCI138745

27. L. Du, Y. He, Y. Zhou, S. Liu, B.-J. Zheng, S. JiangThe spike protein of SARS-CoV--a target for vaccine and therapeutic development

28. Nat Rev Microbiol, 7 (2009), pp. 226236, 10.1038/nrmicro2090

29. P.-R. Hsueh, L.-M. Huang, P.-J. Chen, C.-L. Kao, P.C. YangChronological evolution of $\operatorname{IgM}, \operatorname{IgA}, \operatorname{IgG}$ and neutralisation antibodies after infection with SARSassociated coronavirus

30. Clin Microbiol Infect, 10 (2004), pp. 10621066, 10.1111/j.1469-0691.2004.01009.x

31. G.J. Gorse, M.M. Donovan, G.B. PatelAntibodies to coronaviruses are higher in older compared with younger adults and binding antibodies are more sensitive than neutralizing antibodies in identifying coronavirus-associated illnesses

32. J Med Virol, 92 (2020), pp. 512-517, 10.1002/jmv.25715

33. M. Rokni, V. Ghasemi, Z. TavakoliImmune responses and pathogenesis of SARS-CoV-2 during an outbreak in Iran: Comparison with SARS and MERS

34. Rev Med Virol (2020), 10.1002/rmv.2107
35. Y. Zhang, M. Xiao, S. Zhang, P. Xia, W. Cao, W. Jiang, et al.Coagulopathy and antiphospholipid antibodies in patients with Covid-19

36. N Engl J p. e38, 10.1056/NEJMc2007575

Med, 382 (17) (2020),

37. https://covid19.who.int/?gclid=Cj0KCQjw2or8BRCNARIsA C_ppyYuge-twBBhLm-h7SDeVGGp_mJic0-kXW6nIx5XHg1yHTC9WdrLP0aAlgJEALw wcB

38. https://www.who.int/docs/defaultsource/coronaviruse/situation-reports/20200306-sitrep-46covid-19.pdf?sfvrsn=96b04adf 4

39. Jin Young Ahn, Yujin Sohn,Su Hwan Lee, Use of Convalescent Plasma Therapy in Two COVID-19 Patients with Acute Respiratory Distress Syndrome in Korea

40. J Korean Med Sci, 2020 Apr 13;35(14):e149. doi.org/10.3346/jkms.2020.35.e149

41. Kai Duan, Bende Liu, Ceshend Li, Effectiveness of convalescent plasma therapy in severe Covid-19 patients. 9490-9496,PNAS April 28,2020,vol. 117, no. 17 doi.org/10.3410/f.737695760.793573172

42. Ling Li, Wei Zhang, Yu Hu, Effect of Convalescent Plasma Therapy on Time to Clinical Improvement in Patients With Severe and Life-threatening COVID-19

43. JAMA. 2020;324(5):460-470. doi:10.1001/jama.2020.10044

44. Anup Agarwal, Aparna Mukherjee,Gunjan Kumar; Convalescent plasma in the management of moderate COVID-19 in India: An open-label parallel-arm phase II multicentre randomized controlled trial (PLACID Trial)

45. medRxiv preprint doi: https://doi.org/10.1101/2020.09.03.20187252, September 10 2020.

46. Raymond M Johnson,Joseph M Vinetz, Dexamethasone in the management of covid 19 BMJ 2020; 370 doi: https://doi.org/10.1136/bmj.m2648 (Publ ished 03 July 2020)Cite this as: BMJ 2020;370:m2648

47. John H. Beigel, Kay M. Tomashek, Lori E. Dodd Remdesivir for the Treatment of Covid-19 https://www.nejm.org/doi/full/10.1056/NEJMoa2007764. 